\title{
Surface energy and surface tension of liquid metal nanodrops
}

\author{
M.A. Shebzukhova ${ }^{i}$ and A.A. Shebzukhov \\ Kabardino-Balkarian State University, 360004, Nalchik, Russia
}

\begin{abstract}
A unitary approach has been proposed for the calculation of surface energy and surface tension of nanoparticle being in equilibrium with its saturated vapor on both flat and curved surfaces at given temperature. The final equations involve parameters dependent on the type of premelting structure: bcc, fcc or hep.
\end{abstract}

The surface energy $\bar{u}$ and the surface tension $\sigma$ are the most important thermodynamic characteristics of the layer between the coexistent phases. These quantities, in turn, allow one to obtain other characteristics of the interface boundaries such as work of adhesion, edge angle of wetting, coefficient of spreading etc. The values of $\bar{u}$ and $\sigma$ are of special interest when studying the properties of nanoobjects (particles, bubbles, thin films) because the increase of surface contribution to the properties of the whole system becomes intrinsic.

This communication deals with the calculation of surface energy and surface tension of nanoparticle held in equilibrium with its saturated vapor at constant temperature on the basis of proposed unitary approach.

Let the nanoparticle be sphere of radius $r$ corresponding to the equimolar dividing surface at which the autoadsorption, as known, is nil $\left(\tilde{A}=\bar{N} / \omega_{e}=0\right.$, $\bar{N}=0$ - number of excess particles, $\omega_{e}$ - molar area).

The surface energy and surface tension shall be the excess energy $\bar{u}_{e}$ and excess free energy $\bar{F}_{e}$ per unit area of equimolar dividing surface respectively:

$\bar{u}=\frac{\bar{u}_{e}}{\omega_{e}}=\frac{u-u_{\mathrm{v}}^{(\alpha)} V_{\bar{N}=0}^{(\alpha)}-u_{\mathrm{v}}^{(\beta)} V_{\bar{N}=0}^{(\beta)}}{\omega_{e}}$,

$\sigma=\frac{\bar{F}_{e}}{\omega_{e}}=\frac{F-f_{V}^{(\alpha)} V_{\bar{N}=0}^{(\alpha)}-f_{V}^{(\beta)} V_{\bar{N}=0}^{(\beta)}}{\omega_{e}}$,

where $u$ and $F$ - internal and free energies of the system, $u_{\mathrm{v}}^{(\alpha)}$ and $u_{\mathrm{v}}^{(\beta)}$ - densities of energy in bulk phases $\alpha$ and $\beta$ of volumes $V_{\bar{N}=0}^{(\alpha)}$ and $V_{\bar{N}=0}^{(\beta)}$, located each side from the equimolar dividing surface, $f_{V}^{(\alpha)}$ and $f_{V}^{(\beta)}$ - densities of free energy in mentioned phases.
Let us introduce two more dividing surfaces conditioned by

$$
\begin{aligned}
& \bar{u}=u-u_{\mathrm{v}}^{(\alpha)} V_{\bar{u}=0}^{(\alpha)}-u_{\mathrm{v}}^{(\beta)} V_{\bar{u}=0}^{(\beta)}=0, \\
& \bar{F}=F-f_{\mathrm{v}}^{(\alpha)} V_{\bar{F}=0}^{(\alpha)}-f_{\mathrm{v}}^{(\beta)} V_{\bar{F}=0}^{(\beta)}=0,
\end{aligned}
$$

where $V_{\bar{u}=0}^{(\alpha)}, V_{\bar{u}=0}^{(\beta)}$ and $V_{\bar{F}=0}^{(\alpha)}, V_{\bar{F}=0}^{(\beta)}$ - volumes of phases appearing after the division of all the system by the $\operatorname{surfaces}\left(V=V_{\bar{u}=0}^{(\alpha)}+V_{\bar{u}=0}^{(\beta)}=V_{\bar{F}=0}^{(\alpha)}+V_{\bar{F}=0}^{(\beta)}\right)$.

Simple transformations of (1) and (2) with account of (3) and (4) bring us to

$$
\begin{aligned}
& \bar{u}=\left(u_{\mathrm{v}}^{(\beta)}-u_{\mathrm{v}}^{(\alpha)}\right) \frac{\Delta V_{e u}}{\omega_{e}}, \\
& \sigma=\left(f_{\mathrm{v}}^{(\beta)}-f_{\mathrm{v}}^{(\alpha)}\right) \frac{\Delta V_{e F}}{\omega_{e}},
\end{aligned}
$$

where $\Delta V_{e u}=V_{\bar{N}=0}^{(\alpha)}-V_{\bar{u}=0}^{(\beta)}, \Delta V_{e F}=V_{\bar{N}=0}^{(\alpha)}-V_{\bar{F}=0}^{(\alpha)}$ with

$$
\begin{aligned}
& \Delta V_{e u}=\int_{(V)}\left[\frac{\rho(z)-\rho^{(\beta)}}{\rho^{(\alpha)}-\rho^{(\beta)}}-\frac{u_{\mathrm{v}}(r)-u_{\mathrm{v}}^{(\beta)}}{u_{\mathrm{v}}^{(\alpha)}-u_{\mathrm{v}}^{(\beta)}}\right] d \vec{r}, \\
& \Delta V_{e F}=\int_{(V)}\left[\frac{\rho(z)-\rho^{(\beta)}}{\rho^{(\alpha)}-\rho^{(\beta)}}-\frac{f_{\mathrm{v}}(r)-f_{\mathrm{v}}^{(\beta)}}{f_{\mathrm{v}}^{(\alpha)}-f_{\mathrm{v}}^{(\beta)}}\right] d \vec{r} .
\end{aligned}
$$

$\rho(\mathrm{z}), u_{\mathrm{v}}(r)$ and $f_{\mathrm{v}}(r)$ are molar densities of substance, energy and free energy in transition layer. For spherical nanoparticle in dispersion medium (vapor) we get

$$
\bar{u}=\left(u_{\mathrm{v}}^{(\beta)}-u_{\mathrm{v}}^{(\alpha)}\right) \Delta r_{e u}\left[1-\frac{\Delta r_{e u}}{r_{e}}+\frac{1}{3}\left(\frac{\Delta r_{e u}}{r_{e}}\right)^{2}\right],
$$


$\sigma=\left(f_{\mathrm{v}}^{(\beta)}-f_{\mathrm{v}}^{(\alpha)}\right) \Delta r_{e u}\left[1-\frac{\Delta r_{e F}}{r_{e}}+\frac{1}{3}\left(\frac{\Delta r_{e F}}{r_{e}}\right)^{2}\right]$,

where $\Delta r_{e u}=r_{e}-r_{u}$ and $\Delta r_{e F}=r_{e}-r_{F}$ - distances from the equimolar dividing surface to the separating surfaces corresponding to the conditions of $\bar{u}=0$ and $\bar{F}=0$. Switching to the planar case $\left(r_{e} \rightarrow \infty\right)$ and after some transformations we obtain

$\bar{u}_{\infty}=\frac{\Delta H-R T}{v} \Delta z_{\bar{u}}$

$\sigma_{\infty}=\mu\left(\rho^{(\beta)}-\rho^{(\alpha)}\right) \Delta z_{\sigma}$,

where $\Delta z_{\bar{u}}=\lim _{r_{e u} \rightarrow \infty} \Delta r_{e u}, \Delta z_{\sigma}=\lim _{r_{e u} \rightarrow \infty} \Delta r_{e F}, \Delta H-$ the heat of phase transition $\alpha \rightarrow \beta, \mu$ - chemical potential in the bulk $\left(\mu=\mu^{(\alpha)}=\mu^{(\beta)}\right)$.

The equations (9) and (10) are taken from [1], while (10) and (12) - from [2]. If $\Delta r_{e u}$ and $\Delta r_{e F}$ are supposed to be independent from the radius $r_{e}$ (as Tolman accepted in his formula for $\sigma(r)$ [3]), then it comes from (9)-(12) that

$\bar{u}(r)=\bar{u}_{\infty}\left[1-\frac{\Delta z_{\bar{u}}}{r_{e}}+\frac{1}{3}\left(\frac{\Delta z_{\bar{u}}}{r_{e}}\right)^{2}\right]$,
$\sigma(r)=\sigma_{\infty}\left[1-\frac{\Delta z_{\sigma}}{r_{e}}+\frac{1}{3}\left(\frac{\Delta z_{\sigma}}{r_{e}}\right)^{2}\right]$,

where $\Delta z_{\bar{u}}=\Delta z_{e u}, \quad \Delta z_{\sigma}=\Delta z_{e F}$.

Using (11) and (12) one can, if knows the values of $\bar{u}_{\infty}$ and $\sigma_{\infty}$, calculate $\Delta z_{\bar{u}}$ and $\Delta z_{\sigma}$ and then find dependences for $\bar{u}(r)$ and $\sigma(r)$ according equations (13) and (14). Similar calculations of $\Delta z_{\bar{u}}$ on the basis of experimental data on $\bar{u}_{\infty}$ (obtained from GibbsHelmholtz formula and on measured values of $\sigma$ and $d \sigma / d T$ ) were performed for 20 metals in [1].

Here we present calculation for 50 liquid metals, results for $\Delta z_{\bar{u}}^{(E)}$ being collected in Table 1. The values of $\Delta z_{\bar{u}}^{(E)}$ for the same 50 liquid metals obtained on the basis of experimental data of $\sigma$ are reported in [2] by us.

Now we are going to define the relations for the $\Delta z_{\bar{u}}$ and $\Delta z_{\sigma}$. Following [2] we have

$\Delta z_{\sigma}=B_{\sigma} v^{1 / 3}$

where $\mathrm{v}$ - molar volume of the liquid and the constant $B_{\sigma}$ is $0,128 \cdot 10^{-8}, 0,140 \cdot 10^{-8}$ and $0,204 \cdot 10^{-8}$ for bcc, fcc and hcp - structures of premelting respectively, $B_{\sigma}=0,284 \cdot 10^{-8}$ for liquid $\mathrm{Hg}$ possessing rombohedron structure of premelting.
The value of $\Delta z_{\bar{u}}$ will be found self-consistently availing isotropic model for the liquid metal [4] and using Gibbs-Helmholtz formula $\bar{u}_{\infty}=\sigma_{\infty}\left(1-\frac{T}{\sigma_{\infty}} \frac{d \sigma_{\infty}}{d T}\right)$. The term in round brackets of the latter can be approximately estimated, following [3], as

$1-\frac{T}{\sigma_{\infty}} \frac{d \sigma_{\infty}}{d T} \simeq \frac{L_{0}}{L_{0}-3 R T \ln 2}-\frac{2}{3} \frac{T}{D} \frac{d D}{d T}$,

where $L_{0}$ - heat of evaporation of the overcooled liquid at absolute zero temperature (per mole), $D$ - density (of mass) of liquid metal, $d D / d T$ - temperature coefficient of the density, $R$ - universal gas constant. Now, using (11) and (12) we have

$\Delta z_{\bar{u}}=B_{\bar{u}} \mathrm{v}^{1 / 3}$,

where

$B_{\bar{u}}=B_{\sigma} \frac{\mu}{R T-\Delta H}\left(\frac{L_{0}}{L_{0}-3 R T \ln 2}-\frac{2}{3} \frac{T}{D} \frac{d D}{d T}\right)$.

The values of $\Delta H, D, d D / d T, L_{0}$ and $B_{\sigma}$ entering (18) are known, the chemical potential can be found as $\mu=R T \ln \lambda=R T \ln \left[V_{Q} \cdot P /(k T)\right]$, where $\lambda$ - activity, $P$ - vapor pressure, $V_{Q}=\left[2 \pi \hbar^{2} /\left(a_{0} A k T\right)\right]^{3 / 2}-$ quantum volume, $A$ - atomic mass, $\hbar=h / 2 \pi, k$ - Boltzmann constant, $a_{0}=1,6604 \cdot 10^{-24}$ grams.

We have calculated parameters of $\Delta z_{\bar{u}}$ and $\Delta z_{\sigma}$, values of surface energy $\bar{u}_{\infty}$ and surface tension $\sigma_{\infty}$ and size dependences of $\bar{u}\left(r_{e}\right)$ and $\sigma\left(r_{e}\right)$ at melting point with the above equations for the 50 liquid metals. The bulk properties of liquid metals needed for the calculus were taken from [2]. The required quantities of $\Delta H$, $d D / d T$ and $d \sigma / d T$ for liquid metals (absent in [2]) are given in Table 1 . The values of $L_{0}$ were calculated on the known $\Delta H$ according [4]. The full data for the calculation of values related to the surface tension $\left(\Delta z_{\sigma}, \sigma_{\infty}\right.$ and $\sigma(r))$ are presented in [2] for all metals.

The results of calculations demonstrate (Table 1) that the size parameters $\Delta z_{\bar{u}}$ and $\Delta z_{\sigma}$ are positive in sign for all metals and negligible in absolute measure (they are less than average distance between particles in the liquid). The former reveals that the equimolar surface is farther than rest mentioned dividing surfaces corresponding to the zero excess free energy $(\bar{F}=0)$ and zero cohesion energy $(\bar{u}=0)$. The trifle values of $\Delta z_{\sigma}$ and $\Delta z_{\bar{u}}$ confirm the relevant proximity of the profiles of density and free energy as well as of density and potential energy in surface layer. Besides, the dividing surface corresponding to zero cohesion energy is closer to the condensed phase in comparison to the dividing surface determined by zero excess free energy in the transition layer, the physical boundary liquid-vapor being the same at melting point. 
Table 1. The values of $\Delta z_{\bar{u}}, \Delta z_{\sigma}$, surface energy $\bar{u}_{\infty}$ and surface tension $\sigma_{\infty}$ for the flat boundary liquid metal - vapor at melting point (the given are also input data on $\Delta H, v, d D / d T, d \sigma / d T$ )

\begin{tabular}{|c|c|c|c|c|c|c|c|c|c|c|c|}
\hline $\begin{array}{c}\text { Metal / } \\
\text { premelting } \\
\text { structure }\end{array}$ & $\begin{array}{c}\Delta H, \\
J / \text { mole }\end{array}$ & $\begin{array}{c}v \\
10^{-6}, m^{3}\end{array}$ & $\begin{array}{c}-\frac{d \rho}{d T} \\
\frac{k g}{m^{3} \cdot K}\end{array}$ & $\begin{array}{l}-\frac{d \sigma}{d T}, \\
\frac{m J}{m^{2} \cdot K}\end{array}$ & $\begin{array}{c}\Delta z_{\bar{u}}^{(E)} \\
10^{-10} \mathrm{~m}\end{array}$ & $\begin{array}{c}\Delta z_{\bar{u}}^{(T)}, \\
10^{-10} \mathrm{~m}\end{array}$ & $\frac{\Delta z_{\bar{u}}^{(E)}}{\Delta z_{\sigma}^{(E)}}$ & $\begin{array}{l}\bar{u}_{\infty}^{(E)}, \\
\frac{m J}{m^{2}}\end{array}$ & $\begin{array}{l}\bar{u}_{\infty}^{(T)}, \\
\frac{m J}{m^{2}}\end{array}$ & $\frac{\bar{u}_{\infty}}{\sigma_{\infty}}$ & $\begin{array}{c}\frac{\bar{u}^{(E)}-\bar{u}^{(T)}}{\bar{u}^{(E)}} \\
\times 100 \%\end{array}$ \\
\hline Li bcc & 134686 & 13,221 & 0,052 & 0,150 & 0,475 & 0,421 & 1,386 & 463,1 & 410,98 & 1,104 & 11,25 \\
\hline $\mathrm{Na}$ bcc & 9037,66 & 24,277 & 0,236 & 0,090 & 0,642 & 0,578 & 1,563 & 227,39 & 204,79 & 1,107 & 9,94 \\
\hline $\mathrm{K}$ bcc & 79000 & 47,683 & 0,229 & 0,070 & 0,786 & 0,717 & 1,545 & 125,59 & 114,58 & 1,134 & 8,77 \\
\hline $\mathrm{Rb} b c c$ & 76000 & 58,135 & 0,486 & 0,053 & 0,904 & 0,836 & 1,679 & 103,54 & 95,8 & 1,127 & 7,47 \\
\hline Cs bcc & 65899,58 & 72,228 & 0,638 & 0,045 & 0,942 & 0,926 & 1,734 & 82,64 & 81,26 & 1,145 & 1,66 \\
\hline Be bcc & 294560 & 6,346 & 0,116 & 0,156 & 0,361 & 0,360 & 1,513 & 1603 & 1598 & 1,175 & 0,34 \\
\hline Mg hcp & 127405,86 & 15,286 & 0,265 & 0,150 & 0,928 & 0,967 & 1,751 & 727 & 757,14 & 1,231 & $-4,20$ \\
\hline $\mathrm{Ca}$ bcc & 183920 & 27,640 & 0,221 & 0,062 & 0,629 & 0,642 & 1,662 & 398 & 405,20 & 1,224 & $-1,92$ \\
\hline Sr fcc & 144000 & 35,331 & 0,262 & 0,060 & 0,908 & 0,941 & 2,045 & 348 & 360,32 & 1,205 & $-3,68$ \\
\hline Ba bcc & 150920,51 & 41,352 & 0,526 & 0,07 & 1,002 & 0,996 & 2,254 & 346 & 343,86 & 1,219 & 0,56 \\
\hline $\mathrm{Cu} \mathrm{fcc}$ & 304364 & 7,905 & 0,801 & 0,21 & 0,441 & 0,439 & 1,508 & 1635 & 1628 & 1,163 & 0,43 \\
\hline Ag fcc & 255062 & 11,519 & 0,907 & 0,125 & 0,506 & 0,519 & 1,643 & 1074 & 1103 & 1,161 & $-2,68$ \\
\hline $\mathrm{Au} \mathrm{fcc}$ & 330962,34 & 11,591 & 1,500 & 0,190 & 0,516 & 0,509 & 1,610 & 1424 & 1403 & 1,147 & 1,46 \\
\hline Zn hcp & 114657 & 9,923 & 1,100 & 0,260 & 0,883 & 0,821 & 1,717 & 969,18 & 900,69 & 1,185 & 7,07 \\
\hline Cd hcp & 99874,48 & 14,023 & 1,160 & 0,080 & 0,897 & 0,901 & 1,681 & 607,54 & 610 & 1,162 & $-0,40$ \\
\hline $\mathrm{Al} \mathrm{fcc}$ & 290794,98 & 11,384 & 0,280 & 0,146 & 0,429 & 0,437 & 1,382 & 1066 & 1086 & 1,131 & $-1,81$ \\
\hline Ga bcc & 249372,39 & 11,482 & 0,662 & 0,088 & 0,356 & 0,369 & 1,168 & 764,66 & 793,60 & 1,041 & $-3,79$ \\
\hline In bcc & 219665 & 16,126 & 0,430 & 0,081 & 0,433 & 0,401 & 1,238 & 579,81 & 537,60 & 0,922 & 7,29 \\
\hline $\begin{array}{l}\text { Tl bcc } \\
\text { Tl fcc }\end{array}$ & 173430,96 & 18,071 & 1,430 & 0,080 & $\begin{array}{l}0,561 \\
0,561\end{array}$ & $\begin{array}{l}0,489 \\
0,536\end{array}$ & $\begin{array}{l}1,455 \\
1,455\end{array}$ & $\begin{array}{l}497,1 \\
497,1\end{array}$ & $\begin{array}{c}433,58 \\
474,9\end{array}$ & $\begin{array}{l}1,106 \\
1,107\end{array}$ & $\begin{array}{c}12,77 \\
4,47\end{array}$ \\
\hline $\begin{array}{l}\text { Fe bcc } \\
\text { Fe fcc }\end{array}$ & 341464,43 & 7,757 & 0,883 & 0,230 & $\begin{array}{l}0,540 \\
0,540\end{array}$ & $\begin{array}{l}0,457 \\
0,530\end{array}$ & $\begin{array}{l}1,799 \\
1,799\end{array}$ & $\begin{array}{l}2273 \\
2273\end{array}$ & $\begin{array}{l}1923 \\
2104\end{array}$ & $\begin{array}{l}1,240 \\
1,240\end{array}$ & $\begin{array}{c}15,39 \\
7,40\end{array}$ \\
\hline Co fcc & 355941,42 & 7,312 & 0,988 & 0,340 & 0,533 & 0,480 & 1,746 & 2431 & 2186 & 1,733 & 10,08 \\
\hline $\mathrm{Ni} f \mathrm{fc}$ & 353514,64 & 7,424 & 1,160 & 0,330 & 0,518 & 0,485 & 1,769 & 2340 & 2191 & 1,269 & 6,39 \\
\hline $\mathrm{Rh}$ fcc & 495000 & 9,528 & 0,896 & 0,300 & 0,517 & 0,484 & 1,624 & 2586 & 2420 & 1,222 & 6,43 \\
\hline Ir fcc & 558410,04 & 9,913 & 0,935 & 0,230 & 0,532 & 0,487 & 1,668 & 2876 & 2632 & 1,101 & 8,46 \\
\hline $\mathrm{Pt} \mathrm{fcc}$ & 468619,25 & 10,423 & 2,900 & 0,310 & 0,576 & 0,561 & 1,871 & 2494 & 2433 & 1,282 & 2,46 \\
\hline Ti bcc & 470711,30 & 11,678 & 0,702 & 0,200 & 0,457 & 0,444 & 1,530 & 1778 & 1727 & 1,293 & 2,88 \\
\hline Zr bcc & 581590 & 15,728 & 0,310 & 0,17 & 0,516 & 0,488 & 1,517 & 1732 & 1638 & 1,142 & 5,41 \\
\hline Hf bcc & 661087,87 & 16,080 & 2,318 & 0,210 & 0,567 & 0,611 & 1,946 & 2113 & 2277 & 1,291 & $-7,75$ \\
\hline $\mathrm{V} \mathrm{bcc}$ & 458577,41 & 7,383 & 0,531 & 0,230 & 0,403 & 0,393 & 1,571 & 2404 & 2343 & 1,193 & 2,55 \\
\hline $\mathrm{Nb}$ bcc & 609623,4 & 11,865 & 0,800 & 0,170 & 0,482 & 0,420 & 1,411 & 2305,8 & 2007,3 & 1,026 & 12,95 \\
\hline Ta bcc & 770292,89 & 12,063 & 1,147 & 0,250 & 0,523 & 0,485 & 1,650 & 3222 & 2987 & 1,243 & 7,28 \\
\hline Cr bcc & 392050,21 & 8,112 & 0,300 & 0,200 & 0,521 & 0,480 & 1,847 & 1973 & 1816,8 & 1,207 & 7,90 \\
\hline Mo bcc & 630233,47 & 10,272 & 0,743 & 0,180 & 0,449 & 0,450 & 1,617 & 2651 & 2653 & 1,228 & $-0,10$ \\
\hline $\mathrm{W} \mathrm{bcc}$ & 823849,37 & 11,349 & 1,250 & 0,210 & 0,459 & 0,475 & 1,680 & 3297 & 3415 & 1,226 & $-3,58$ \\
\hline $\mathrm{Mn} \mathrm{fcc}$ & 243514,64 & 9,066 & 0,700 & 0,200 & 0,646 & 0,578 & 1,928 & 1393 & 1246 & 1,228 & 10,59 \\
\hline Re bcc & 732217,57 & 9,852 & 0,800 & 0,180 & 0,477 & 0,461 & 1,671 & 3403 & 3294 & 1,133 & 3,20 \\
\hline Sn bcc & 230125,52 & 17,179 & 0,194 & 0,160 & 0,509 & 0,494 & 1,496 & 670 & 649 & 1,049 & 3,04 \\
\hline $\mathrm{Pb} \mathrm{fcc}$ & 177824,27 & 19,097 & 1,317 & 0,085 & 0,559 & 0,552 & 1,477 & 506 & 500 & 1,104 & 1,20 \\
\hline Bi bcc & 170962,34 & 20,938 & 1,200 & 0,0766 & 0,524 & 0,491 & 1,394 & 417 & 390 & 1,099 & 6,42 \\
\hline $\mathrm{Hg}$ rhomb & 59297,07 & 14,749 & 2,400 & 0,281 & 1,447 & 1,456 & 1,394 & 563 & 566 & 1,095 & $-0,58$ \\
\hline $\mathrm{Ru} \mathrm{fcc}$ & 527447,7 & 9,272 & 2,146 & 0,310 & 0,555 & 0,611 & 2,078 & 3032 & 3337 & 1,413 & $-10,04$ \\
\hline Tb bcc & 356900 & 21,950 & 0,152 & 0,060 & 0,510 & 0,563 & 1,572 & 797,8 & 880,7 & 1,104 & $-10,38$ \\
\hline Os bcc & 748954 & 9,463 & 3,655 & 0,330 & 0,471 & 0,483 & 1,949 & 3589 & 3685 & 1,354 & $-2,67$ \\
\hline Ce bcc & 350000 & 20,96 & 0,227 & 0,070 & 0,541 & 0,520 & 1,469 & 880 & 846 & 1,078 & 3,84 \\
\hline Pr bcc & 296652,7 & 21,314 & 0,240 & 0,080 & 0,597 & 0,547 & 1,544 & 802,4 & 735,25 & \begin{tabular}{|l|}
1,101 \\
\end{tabular} & 8,37 \\
\hline $\mathrm{Nd} \mathrm{bcc}$ & 317782 & 21,567 & 0,528 & 0,065 & 0,541 & 0,545 & 1,531 & 771 & 776 & 1,137 & $-0,71$ \\
\hline Gd bcc & 305000 & 22,024 & 0,793 & 0,060 & 0,698 & 0,738 & 2,062 & 925 & 978 & 1,208 & $-5,74$ \\
\hline Th bcc & 514000 & 22,099 & 1,847 & 0,140 & 0,618 & 0,641 & 1,919 & 1391 & 1442 & 1,207 & $-3,63$ \\
\hline U bcc & 420000 & 13,298 & 1,031 & 0,140 & 0,537 & 0,500 & 1,634 & 1650 & 1534 & 1,111 & 7,04 \\
\hline La bcc & 382845 & 23,326 & 0,237 & 0,110 & 0,548 & 0,515 & 1,407 & 876 & 823,5 & 1,088 & 6,03 \\
\hline
\end{tabular}

Calculations show that $\Delta z_{\bar{u}}>\Delta z_{\sigma}$ all the time what tells that the size effects of surface energy start emerging for bigger particles compared to the surface tension.
The results show that $\Delta z_{\bar{u}}$, and also $\Delta z_{\sigma}$, change "inshape" with the molar volume and become larger with increasing temperature. 
The latter can be associated, as mentioned in [1], to the increasing diffusion and thickness of the transition layer when heating liquid metal. It comes from Table 1 that calculated values of surface energy $\bar{u}_{\infty}^{(T)}$ and surface tension $\sigma_{\infty}^{(T)}$ of liquid metals appearing on flat boundary surrounded by saturated vapor at melting point agree well with the most reliable experimental data for all 50 elements. The values of $\bar{u}_{\infty}^{(E)}$ were calculated on the measured data of $\sigma_{\infty}^{(E)}$ and $d \sigma^{(E)} / d T$. The parameters of $\Delta z_{\bar{u}}$, obtained by theory, $\Delta z_{\bar{u}}^{(T)}$, and by experiment, $\Delta z_{\bar{u}}^{(E)}$, agree quite well too.

The calculations of size dependence $\bar{u}(r)$ and $\sigma(r)$ were carried out in range from $r_{e \min }=\Delta z_{\bar{u}}$ and $r_{e \text { min }}=\Delta z_{\sigma}$ to $r_{e}=\infty$ respectively. One can easily see that the relations $\bar{u}\left(r_{e \min }\right)=\bar{u}_{\infty} / 3$ and $\sigma\left(r_{\text {emin }}\right)=\sigma_{\infty} / 3$ are valid at given minimal radii $r_{e \text { min }}$. The calculations for the smaller sizes are out of matter due to the negligibility of $\Delta z_{\bar{u}}$ and $\Delta z_{\sigma}$, see Table 1 (they are less than 10 nanometers except for the mercury), and, the more, there is no reason to ignore the dependence of $\Delta z_{\bar{u}}$ and $\Delta z_{\sigma}$ on radius (as assumed afore) in case of extremely curved boundaries.
Calculations show that $\bar{u}\left(r_{e}\right)$ and $\sigma\left(r_{e}\right)$ monotonically decrease with decreasing radius of the particle $\left(d \bar{u} / d r_{e}>0, d \sigma / d r_{e}>0\right)$ in mentioned ranges of sizes. The same comes from the calculated data of Table 2 for some liquid metals. The similar data for the surface tension of some other liquid metals were published in [2].

So, equations obtained here allow one to calculate the surface energy $\bar{u}$ and the surface tension $\sigma$ of liquid metals on flat as well as curved surfaces within the unitary approach. The final equations for $\bar{u}$ and $\sigma$ involve parameters $B_{\bar{u}}$ and $B_{\sigma}$ dependent on type of premelting structure being either bcc, fcc or hcp.

\section{References}

1. A.I. Rusanov, V.A. Prohorov, Interface tensiometry (Sankt - Petersburg, Khimia, 1994) [in Russian]

2. M.A. Shebzukhova,Z.A. Shebzukhov, A.A. Shebzukhov, Bulletin of the Russian Academy of Sciences: Physics , 74(5), 697-704 (2010)

3. R.C. Tolman, J. Chem. Phys., 17(2), 333-340 (1949)

4. Q. Jiang, H.M. Lu // Surf. Sci. Rep., 63, 427-464 (2008)

Table 2. The size dependence of the surface energy $\bar{u}\left(r_{e}\right)$ and of surface tension $\sigma\left(r_{e}\right)$ of liquid metals at melting point

\begin{tabular}{|c|c|c|c|c|c|c|c|c|c|c|}
\hline \multirow{2}{*}{$r_{e}, n m$} & \multicolumn{7}{|c|}{$\bar{u}\left(r_{e}\right), \quad m J / m^{2}$} & \multicolumn{5}{|c|}{$\sigma\left(r_{e}\right), \quad m L / m^{2}$} \\
\cline { 2 - 11 } & $\mathrm{K}$ & $\mathrm{Rb}$ & $\mathrm{Au}$ & $\mathrm{Pt}$ & $\mathrm{Mo}$ & $\mathrm{K}$ & $\mathrm{Rb}$ & $\mathrm{Au}$ & $\mathrm{Pt}$ & $\mathrm{Mo}$ \\
\hline 0,1 & 52,74 & 38,14 & 815,60 & 1333,27 & 1638,85 & 61,57 & 50.06 & 852,23 & 1366,75 & 1599,68 \\
0,5 & 106,88 & 85,95 & 1282,10 & 2217,72 & 2420,07 & 92,71 & 78.40 & 1100,75 & 1752,78 & 2015,41 \\
1 & 115,98 & 94,46 & 1351,79 & 2353,10 & 2533,75 & 97,28 & 82.62 & 1135,02 & 1805,85 & 2072,17 \\
2 & 120,72 & 98,93 & 1387,58 & 2422,86 & 2591,93 & 99,62 & 84.79 & 1152,42 & 1832,79 & 2100,95 \\
3 & 122,34 & 100,45 & 1399,65 & 2446,42 & 2611,52 & 100,41 & 85.52 & 1158,26 & 1841,83 & 2110,61 \\
4 & 123,14 & 101,22 & 1405,71 & 2458,26 & 2621,35 & 100,81 & 85.89 & 1161,19 & 1846,36 & 2115,44 \\
5 & 123,63 & 101,68 & 1409,35 & 2465,38 & 2627,27 & 101,04 & 86.11 & 1162,95 & 1849,08 & 2118,35 \\
10 & 124,61 & 102,61 & 1416,66 & 2479,66 & 2639,11 & 101,52 & 86.56 & 1166,47 & 1854,54 & 2124,17 \\
20 & 125,10 & 103,07 & 1420,33 & 2486,82 & 2645,05 & 101,76 & 86.78 & 1168,23 & 1857,27 & 2127,08 \\
30 & 125,26 & 103,23 & 1421,55 & 2489,21 & 2647,03 & 101,84 & 86.85 & 1168,82 & 1858,18 & 2128,06 \\
40 & 125,34 & 103,31 & 1422,16 & 2490,41 & 2648,03 & 101,88 & 86.89 & 1169,12 & 1858,63 & 2128,54 \\
50 & 125,39 & 103,35 & 1422,53 & 2491,13 & 2648,62 & 101,90 & 86.91 & 1169,29 & 1858,91 & 2128,83 \\
100 & 125,49 & 103,45 & 1423,27 & 2492,56 & 2649,81 & 101,95 & 86.96 & 1169,65 & 1859,45 & 2129,42 \\
200 & 125,54 & 103,49 & 1423,63 & 2493,28 & 2650,40 & 101,98 & 86.98 & 1169,82 & 1859,73 & 2129,71 \\
$\infty$ & 125,59 & 103,54 & 424,00 & 2494,00 & 2651,00 & 102,00 & 87,00 & 1170,00 & 1860,00 & 2130,00 \\
\hline
\end{tabular}

\footnotetext{
${ }^{\mathrm{i}}$ Author to whom all the correspondence should be sent, e-mail: sh-madina@mail.ru
} 